\title{
$\mathrm{N}$-Acetylcysteine inhibits platelet-monocyte conjugation in patients with type 2 diabetes with depleted intraplatelet glutathione: a randomised controlled trial
}

\author{
A. T. Treweeke • T. J. Winterburn • I. Mackenzie • \\ F. Barrett • C. Barr • G. F. Rushworth • I. Dransfield • \\ S. M. MacRury • I. L. Megson
}

Received: 4 May 2012 /Accepted: 9 July 2012 /Published online: 31 August 2012

(C) The Author(s) 2012. This article is published with open access at Springerlink.com

\begin{abstract}
Aims/hypothesis The aim of this study was to determine whether oral dosing with $N$-acetylcysteine (NAC) increases intraplatelet levels of the antioxidant, glutathione (GSH), and reduces platelet-monocyte conjugation in blood from patients with type 2 diabetes.

Methods In this placebo-controlled randomised crossover study, the effect of oral NAC dosing on platelet-monocyte conjugation and intraplatelet GSH was investigated in
\end{abstract}

Electronic supplementary material The online version of this article (doi:10.1007/s00125-012-2685-z) contains peer-reviewed but unedited supplementary material, which is available to authorised users.

A. T. Treweeke · T. J. Winterburn • I. Mackenzie •

I. L. Megson ( $\square)$

Free Radical Research Facility, Department of Diabetes \&

Cardiovascular Science, University of the Highlands \& Islands,

Centre for Health Science,

Inverness IV2 3JH, UK

e-mail: ian.megson@uhi.ac.uk

F. Barrett · G. F. Rushworth

NHS Highland, Highland Clinical Research Facility, University of the Highlands \& Islands, Centre for Health Science,

Inverness, UK

C. Barr $\cdot$ S. M. MacRury

Highland Clinical Research Facility, University of the Highlands \& Islands, Centre for Health Science,

Inverness, UK

I. Dransfield

MRC Centre for Inflammation Research,

Queen's Medical Research Institute, University of Edinburgh,

Edinburgh, UK

\section{S. M. MacRury}

Department of Diabetes and Cardiovascular Science,

University of the Highlands \& Islands, Centre for Health Science, Inverness, UK patients with type 2 diabetes (eligibility criteria: men or post-menopausal women with well-controlled diabetes $\left(\mathrm{HbA}_{1 \mathrm{c}}<10 \%\right)$, not on aspirin or statins). Patients ( $n=14$; age range 43-79 years, $\left.\mathrm{HbA}_{1 \mathrm{c}}=6.9 \pm 0.9 \%[52.3 \pm 10.3 \mathrm{mmol} / \mathrm{mol}]\right)$ visited the Highland Clinical Research Facility, Inverness, UK on day 0 and day 7 for each arm of the study. Blood was sampled before and $2 \mathrm{~h}$ after oral administration of placebo or NAC $(1,200 \mathrm{mg})$ on day 0 and day 7. Patients received placebo or NAC capsules for once-daily dosing on the intervening days. The order of administration of NAC and placebo was allocated by a central office and all patients and research staff involved in the study were blinded to the allocation until after the study was complete and the data fully analysed. The primary outcome for the study was platelet-monocyte conjugation.

Results Oral NAC reduced platelet-monocyte conjugation (from $53.1 \pm 4.5 \%$ to $42.5 \pm 3.9 \%$ ) at $2 \mathrm{~h}$ after administration and the effect was maintained after 7 days of dosing. Intraplatelet GSH was raised in individuals with depleted GSH and there was a negative correlation between baseline intraplatelet GSH and platelet-monocyte conjugation. There were no adverse events.

Conclusions/interpretation The NAC-induced normalisation of intraplatelet GSH, coupled with a reduction in platelet-monocyte conjugation, suggests that NAC might help to reduce atherothrombotic risk in type 2 diabetes.

Funding: Chief Scientist Office (CZB/4/622), Scottish Funding Council, Highlands \& Islands Enterprise and European Regional Development Fund.

Trial registration: isrctn.org ISRCTN89304265

Keywords $N$-acetylcysteine $\cdot$ Antioxidative treatment . Atherothrombosis · Glutathione - Monocyte - Platelet . Therapeutics · Type 2 diabetes 


$\begin{array}{ll}\text { Abbreviations } \\ \text { eGFR } & \text { Estimated GFR } \\ \text { NAC } & N \text {-acetylcysteine } \\ \text { NO } & \text { Nitric oxide } \\ \text { PAI-1 } & \text { Plasminogen activator inhibitor-1 } \\ \text { ROS } & \text { Reactive oxygen species } \\ \text { SBD-F } & \text { 7-Fluoro-2,1,3-benzoxadiazole-4-sulfonate } \\ \text { tGSH } & \text { Total glutathione }\end{array}$

\section{Introduction}

Oxidative stress is implicated in both the aetiology and the cardiovascular complications of type 2 diabetes [1-5]. Increased reactive oxygen species (ROS) generation is a feature of platelets in type 2 diabetes [6] and contributes to platelet hyperaggregability associated with the disease [7, 8]. Oxidative stress is also implicit in endothelial dysfunction associated with atherosclerosis and subsequent thrombotic complications [9]. Since the realisation that aspirin fails to show sufficient clinical benefit in primary prevention of cardiovascular events in diabetes to merit its universal prescription to this group of patients $[10,11]$, there is renewed urgency in finding alternatives. Given the central role of oxidative stress in both diabetes and the resultant cardiovascular complications, antioxidant intervention is a promising therapeutic option that could specifically target atherothrombotic processes.

Glutathione (GSH) is an abundant, key endogenous antioxidant that is depressed in platelets from patients with type 2 diabetes, contributing to hyperaggregability [12]. Intramuscular GSH administration increases levels of protective nitric oxide (NO) in platelets from patients with type 2 diabetes, with a concomitant decrease in plasma levels of the fibrinolytic inhibitor, plasminogen activator inhibitor-1 (PAI-1) [13]. However, GSH is a poor candidate for oral therapy because of peptide digestion in the stomach and poor membrane penetration. $\mathrm{N}$-acetylcysteine (NAC), is a well-recognised intravenous therapy used to redress acute GSH depletion in overdosage with paracetamol (known as acetaminophen in the USA and Canada) [14]. NAC is currently under investigation for its antioxidant benefits in a range of clinical conditions [15-20], although there are some doubts about its efficacy, particularly in contrast-induced nephropathy [21].

In the diabetes arena, oral NAC has been shown to improve endothelial function in a rat model of diabetes [22] and to reduce endothelial activation, oxidative stress markers [23] and blood pressure [24] in patients with type 2 diabetes. Very high concentrations ( $3 \mathrm{mmol} / \mathrm{l})$ augment NOmediated inhibition of platelet aggregation in blood from obese patients in vitro [25]. Our own previous studies in vitro indicated that much lower concentrations of NAC, which are achievable with oral dosing (10-100 $\mu \mathrm{mol} / \mathrm{l})$, inhibit platelet function in blood from healthy volunteers [26] and in patients with type 2 diabetes [27]. This effect

was associated with increased intraplatelet GSH, inhibition of oxidative stress and increased levels of NO metabolites.

Here, we tested the hypothesis that oral NAC dosing causes acute $(2 \mathrm{~h})$ inhibition of platelet-monocyte conjugation and microparticle count - both recognised as markers of cardiovascular risk - in blood from patients with type 2 diabetes, and that the effect is maintained after daily dosing for 7 days in free-living individuals. Furthermore, we sought to explore the association between the effectiveness of NAC and baseline intraplatelet GSH.

\section{Methods}

Study participants Eligible patients with type 2 diabetes (Tables 1 and 2) were recruited through local general practitioners. All patients gave written informed consent in line with International Conference on Harmonisation Good Clinical Practice guidelines. At an initial screening visit, venous blood $(9 \mathrm{ml})$ was collected from the antecubital fossa into lithium heparin-containing tubes (Sarstedt, Leicester, UK) to screen lipid and renal status (Piccolo Lipid and Renal Panels, respectively; Abaxis, Darmstadt, Germany); a fingerprick blood sample was obtained for $\mathrm{HbA}_{1 \mathrm{c}}$ determination (DCA 2000+ Analyser; Bayer, Newbury, UK). Individuals meeting the study inclusion criteria returned within 2 weeks for commencement of the clinical study.

Inclusion criteria for the study were as follows: (1) men or post-menopausal women with type 2 diabetes (to avoid the variability of platelet count and behaviour during the menstrual cycle); (2) those not receiving aspirin (aspirin was withdrawn at least 10 days in advance of the first visit and throughout the study period); (3) those not receiving lipid-lowering therapywhere necessary, statins were withdrawn 1 month in advance of the first visit and throughout the study period.

Exclusion criteria for the study were as follows: (1) very poorly controlled diabetes $\left(\mathrm{HbA}_{1 \mathrm{c}}>10 \%\right.$ [86 mmol/mol] $) ;(2)$ significant hypertriacylglycerolaemia (triacylglycerols $>4 \mathrm{mmol} / \mathrm{l}$ ); (3) renal disease, defined by estimated GFR (eGFR) $<40 \mathrm{ml} / \mathrm{min}$; (4) current or recently stopped (within last 6 months) smokers; (5) patients receiving other antiplatelet therapy (e.g. clopidogrel, dipyridamole) or lipid-lowering therapy, or where patients were unable or unwilling to stop

Table 1 Patient characteristics

Data are expressed as mean $\pm \mathrm{SD}(n=14)$

\begin{tabular}{ll}
\hline Characteristic & \\
\hline Age (years) & $63.9 \pm 10.1$ \\
Sex (male/female) & $9: 5$ \\
$\mathrm{BMI}\left(\mathrm{kg} / \mathrm{m}^{2}\right)$ & $33.1 \pm 5.7$ \\
$\mathrm{HbA}_{1 \mathrm{c}}(\%)$ & $6.9 \pm 0.90$ \\
$\mathrm{HbA}_{1 \mathrm{c}}(\mathrm{mmol} / \mathrm{mol})$ & $52.3 \pm 10.3$ \\
$\mathrm{Cholesterol}(\mathrm{mmol} / \mathrm{l})$ & $4.7 \pm 1.1$ \\
\hline
\end{tabular}


Table 2 Clinical management regimens for study patients

\begin{tabular}{ll}
\hline Treatment & No. of patients \\
\hline Diet alone & 4 \\
Metformin alone & 3 \\
Metformin + sulfonylurea & 3 \\
Metformin + sulfonylurea+glitazone & 3 \\
Sulfonylurea alone & 1 \\
\hline
\end{tabular}

therapy for the duration of study participation; (6) current use of tetracycline antibiotics or cough suppressants; (7) patients with asthma, because of the low but increased risk of bronchospasm and the increased frequency of anaphylactoid reactions to NAC in asthma suffers.

\section{Study design}

Patients were randomised to receive either NAC $(1,200 \mathrm{mg})$ or placebo daily for a total of 8 days: both researchers and patients were blinded in this crossover study. On day 0 , patients attended the Highland Clinical Research Facility between 09:00 and 09:30 hours, after an overnight fast to minimise diet-induced and diurnal variations in platelet reactivity. A baseline venous blood sample $(90 \mathrm{ml})$ was drawn from the antecubetal fossa using a $21 \mathrm{G}$ butterfly device and syringe before oral administration of NAC $1,200 \mathrm{mg}(2 \times 600 \mathrm{mg}$ capsules) or placebo. The butterfly was flushed with normal saline after initial sampling and $2 \mathrm{~h}$ later, having discarded the first $2 \mathrm{ml}$ blood, a second blood sample was taken in the same manner as the first and the patients were provided with a 6-day supply of NAC or placebo capsules for once-daily selfadministration. On day 7 of the study, patients returned after an overnight fast for a blood withdrawal before and at $2 \mathrm{~h}$ after their final NAC or placebo dose in this arm of the study (protocol identical to day 0). Patients received a light breakfast of buttered toast and water immediately after baseline measures on study visits. A washout period of at least 1 week was allowed before patients returned for the crossover, which followed the same protocol as the first arm of the study. The study design is summarised in Fig. 1 and the recruitment, enrolment and randomisation process is summarised in electronic supplementary material (ESM) Fig. 1.

Ethics approval Clinical Trial Authorisation was granted by Medicines \& Healthcare Products Regulatory Agency, Eudract number 2008-001620 and the study was approved by the North of Scotland Research Ethics Committee, study number 06/S0901/39. Clinical trial registration number: ISRCTN89304265.

Blood samples Consistent blood sampling and handling is essential for meaningful results for the proposed flow cytometry assays in particular. Drawn blood was rapidly transferred to tubes containing EDTA, trisodium citrate or heparin (Monovette) and gently inverted several times to mix. Heparinised blood was used for platelet-monocyte conjugation and microparticle experiments, citrated plasma for PAI-1 measures and EDTA for platelet total GSH (tGSH) measurement.

Platelet-monocyte conjugation Platelet-monocyte conjugates were measured by flow cytometry detection of dualstained, whole blood samples. Briefly, $100 \mu \mathrm{l}$ of heparinised whole blood was incubated with CD14-FITC and CD41-PECy5 antibodies, or the appropriate isotype controls, for $15 \mathrm{~min}$. After addition of $1 \mathrm{ml} \mathrm{FACS} \mathrm{lysing} \mathrm{solution} \mathrm{(Becton} \mathrm{Dickinson,}$ Oxford, UK), the cells were incubated for a further $15 \mathrm{~min}$ and then immediately analysed for conjugates using a FACSCalibur flow cytometer (Becton Dickinson). Platelet-monocyte conjugates were identified as CD14/CD41-positive events.

Microparticles Microparticles were prepared from heparinised whole blood. Samples were centrifuged at $500 \mathrm{~g}$ for $5 \mathrm{~min}$, the plasma was removed and centrifuged at $5,000 \mathrm{~g}$ for $5 \mathrm{~min}$; this was repeated twice to remove any remaining cells. Next, $1 \mathrm{ml}$ of this plasma was further centrifuged at $16,000 \mathrm{~g}$ for $5 \mathrm{~min}$, and the microparticle pellet was washed with filtered FACS flow (Becton Dickinson), centrifuged again at 16,000 $g$ and then resuspended in $0.5 \mathrm{ml}$ FACS flow spiked with $3 \mu \mathrm{m}$ polystyrene latex beads (Sigma Aldrich, Poole, UK). These purified microparticles were acquired through a FACSCalibur flow cytometer and their numbers calculated relative to the known, fixed number of beads acquired at the same time.

PAI-1 antigen and activity Plasma was prepared by centrifugation of citrated blood at $1,000 \mathrm{~g}$ at room temperature for $10 \mathrm{~min}$ and then frozen at $-70^{\circ} \mathrm{C}$. PAI- 1 antigen and PAI-1 activity were both measured using Zymutest ELISA assay kits (Hyphen BioMed, Paris, France) according to the manufacturer's instructions.

tGSH determination tGSH was measured in platelet extracts using an established HPLC method. Briefly, platelet suspensions (normalised to $150 \times 10^{3} / \mu \mathrm{l}$ ) were lysed before disulfide reduction, achieved by the addition of reducing agent $(10 \%$ tri-butyl phosphine in dimethylformamide; $4^{\circ} \mathrm{C}$ for $30 \mathrm{~min}$ ). Samples were deproteinised by the addition of $150 \mu \mathrm{l}$ trichloroacetic acid (10\% in $1 \mathrm{mmol} / \mathrm{l} \mathrm{EDTA})$, vortexed $(20 \mathrm{~s})$, then centrifuged $\left(8,000 \mathrm{~g}, 5 \mathrm{~min}, 4^{\circ} \mathrm{C}\right)$. One-hundred microlitres of the supernatant fraction was prepared for derivatisation by the addition of $20 \mu \mathrm{l}$ of $1.55 \mathrm{~mol} / \mathrm{l}$ sodium hydroxide, $250 \mu \mathrm{l}$ of $0.125 \mathrm{~mol} / \mathrm{l}$ borate buffer $(\mathrm{pH} 9.5)$ and $100 \mu \mathrm{l}$ of $1 \mathrm{mg} / \mathrm{ml}$ ammonium 7-fluoro-2,1,3-benzoxadiazole-4-sulfonate (SBD-F). Samples were vortexed briefly and incubated $\left(60^{\circ} \mathrm{C}, 1 \mathrm{~h}\right)$. Cooled samples were analysed by HPLC (Agilent 1200 series; Agilent Technologies, Stockport, UK) using 
Fig. 1 Diagrammatic representation of the study design

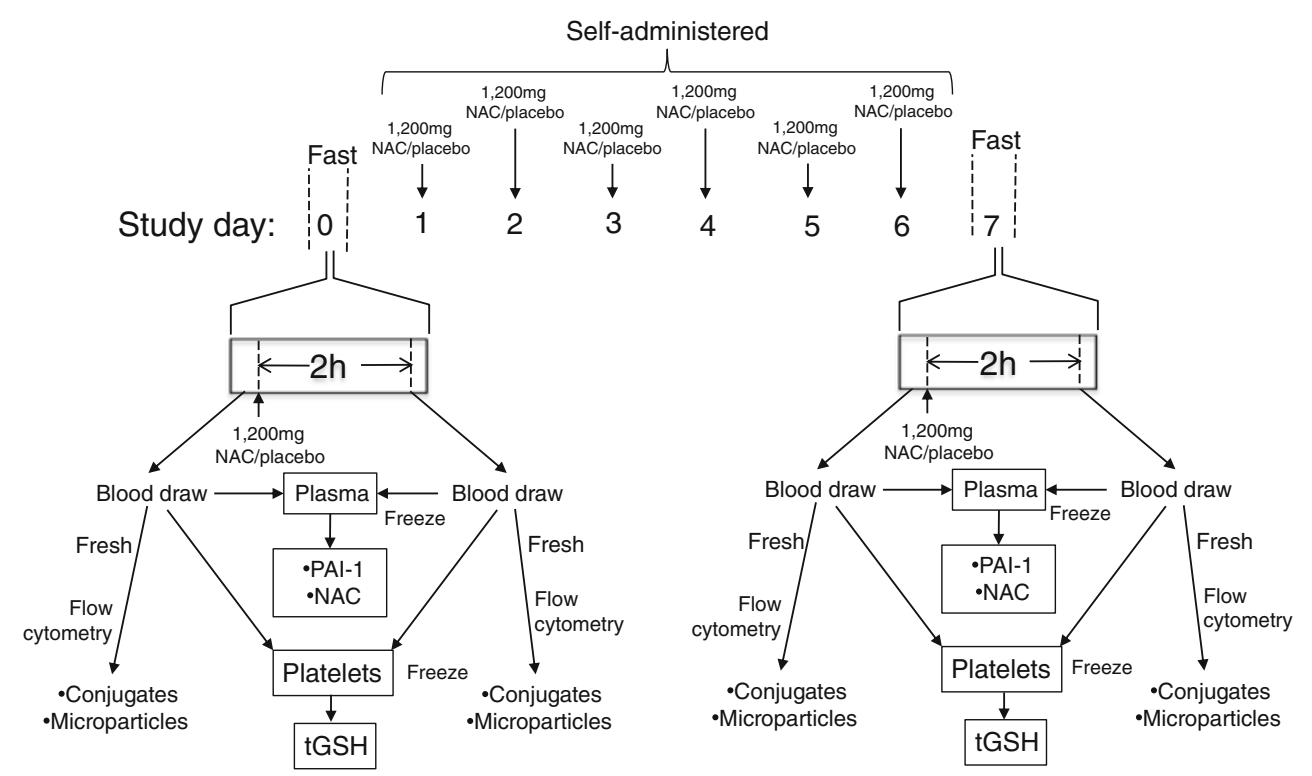

$\geq 1$ week washout before crossover fluorescence detection (excitation $\lambda=385 \mathrm{~nm}$, emission $\lambda=515$ $\mathrm{nm})$. Chromatographic separations were achieved with a Gemini-NX C18 column (250 mm $\times 4.6 \mathrm{~mm} \times 5 \mu \mathrm{m}$; Phenomenex, Macclesfield, UK) and a mobile phase of $7 \%$ acetonitrile, $93 \% 100 \mathrm{mmol} / 1 \mathrm{KH}_{2} \mathrm{PO}_{4}$ at $\mathrm{pH} 2.1$. Forty-microlitre samples were injected onto the column and eluted at a flow rate of $1 \mathrm{ml} /$ min. Thiol derivatives were quantified using peak areas. Protein concentrations in extracts were quantified using a Coomassie protein assay (Perbio Science, Cramlington, UK).

Plasma NAC measurement Total NAC (i.e. oxidised+ reduced) was measured in deproteinised plasma using HPLC with fluorescence detection (as above) according to a method adapted from that of Wilkinson et al [28].

Statistical power A review of the literature pertaining to the various assays conducted in this project identified plateletmonocyte conjugation as the least powerful technique used in this study. The power calculation was therefore conducted for this measure: for a significance level of $p=0.01$ and a power of $80 \%$ for detecting a change in platelet-monocyte conjugation of $10 \%, 15$ individuals were recruited to the study.

Statistical analyses Patient characteristics are expressed as mean $\pm \mathrm{SD}$; all other results are expressed as mean $\pm \mathrm{SEM}$. Curve fitting and statistical tests were performed using GraphPad Prism software (version 5.00; Graphpad, San Diego, CA, USA). Data distribution was assessed using the Kolmogorov-Smirnov test. All data sets followed Gaussian distribution. It was therefore appropriate to use parametric statistics: $t$ tests, two-way ANOVA with repeated measures and Pearson's correlation tests were used, as appropriate. $p<0.05$ was considered to be statistically significant.

Drugs, chemicals, reagents and other materials The investigational product (NAC) and placebo tablets were supplied by Tayside Pharmaceuticals (Dundee, UK). CD14-FITC (BD 555397), IgG2a-FITC (BD 555573), CD41-PE-Cy5 (BD 559768), IgG1-PE-Cy5 (BD 555750) and FACS lysing solution were all obtained from Becton Dickinson. Polystyrene latex beads $(3 \mu \mathrm{m})$ were purchased from Sigma Aldrich (Poole, UK). ELISA kits for the measurement of PAI-1 antigen and activity were from Hyphen BioMed (Paris, France).

\section{Results}

Participant characteristics The characteristics of the study population are shown in Tables 1 and 2. One patient reported feeling nauseous at times between the two visits on the NAC arm of the study but no other adverse reactions were noted. It was not possible to obtain a blood sample from one of the patients after placebo administration at their first visit so data from this patient was excluded from the majority of the analyses. There was insufficient platelet extract for GSH analysis in samples from two further patients.

Platelet-monocyte conjugation Platelet-monocyte conjugation was significantly inhibited in patients at $2 \mathrm{~h}$ after administration of NAC (conjugated monocytes reduced from $53.1 \pm 4.5$ to $42.5 \pm 3.9 \%$ of monocyte population: $p=0.011$; Fig. 2a); no significant effect was seen in the equivalent 

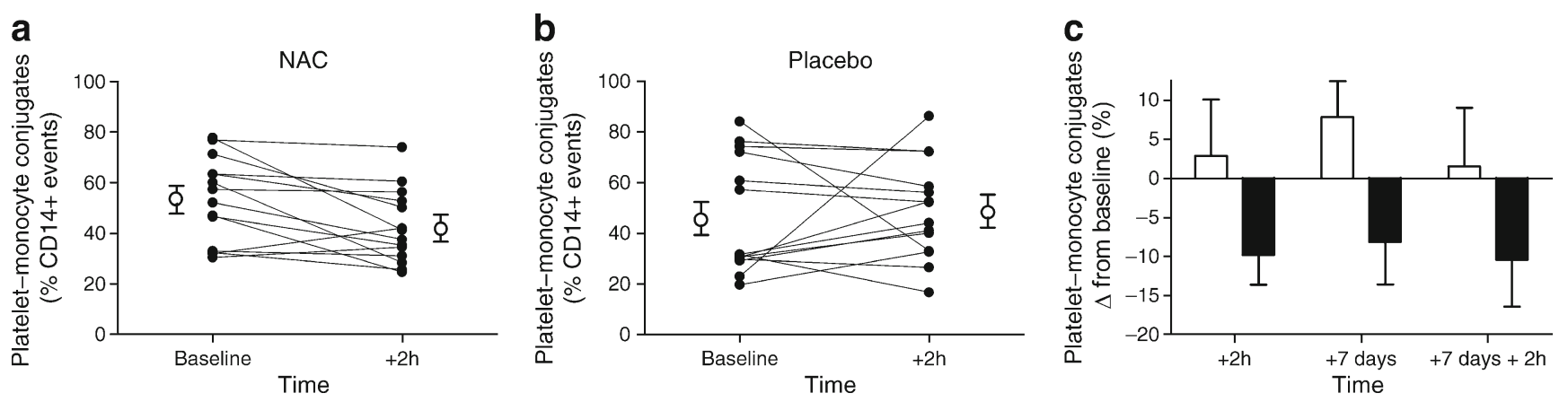

Fig. 2 Effect of NAC (a; $p=0.011 ; n=14$ by paired Student's $t$ test) and placebo $(\mathbf{b} ; p>0.05)$ on platelet-monocyte conjugation after $2 \mathrm{~h}$ and after 7 days of daily oral dosing (c; $p=0.010$ for NAC vs placebo; $n=13$ by repeated measures two-way ANOVA). NAC, but not placebo, was found to reduce platelet-monocyte conjugation at $2 \mathrm{~h}$ after oral

results from the placebo arm of the study ( $p>0.05$; Fig. $2 b)$. Baseline-subtracted data indicate that depression of plateletmonocyte conjugation was maintained (approximately -10 to $-15 \%$ compared with placebo) on day 7 of once-daily administration of $\mathrm{NAC}$, both before and after the final administration (Fig. 2c; $p=0.035$; two-way ANOVA).

Microparticles In the NAC arm of the study there was a trend towards depression of plasma-borne microparticles that did not reach significance $(-31 \% ; p=0.094$; Fig. 3a); microparticles were significantly $(+25 \% ; p=0.048)$ elevated at $2 \mathrm{~h}$ after placebo administration (Fig. 3b).Baseline-subtracted data indicate that this pattern of effect was maintained at day 7 after daily oral dosing; the depression of microparticles in the NAC arm compared with the placebo arm of the study was significant (Fig. 3c; $p<0.016$ ).

Plasma NAC Plasma NAC was only detectable in $8 / 14$ patients $(1-2 \mu \mathrm{mol} / \mathrm{l})$ at $2 \mathrm{~h}$ after NAC administration. No residual NAC was measured at baseline on the second visit (i.e. $\sim 24 \mathrm{~h}$ after the final self-administered dose; threshold for detection, $1 \mu \mathrm{mol} / \mathrm{l})$.

a

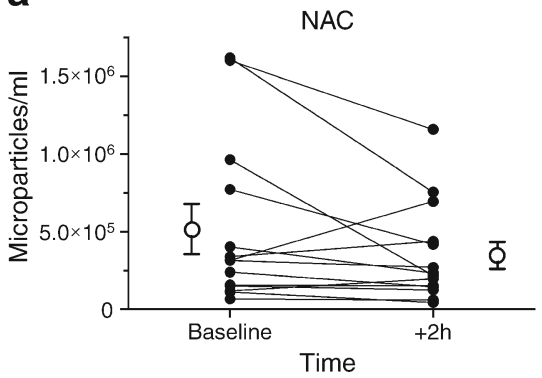

b

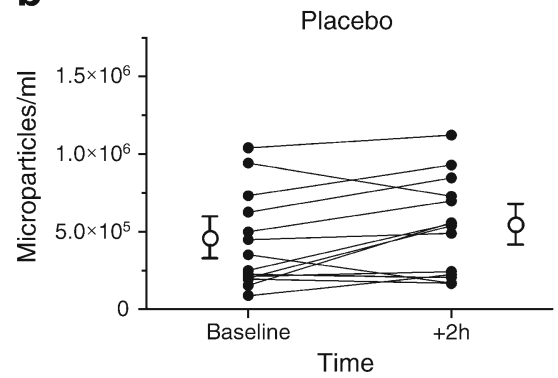

dosing and the reduction compared with placebo was significant across the 7 day dosing period (black bars, NAC; white bars, placebo.) Insufficient blood was drawn from one patient at one visit and data from that patient was excluded from (c)

Plasma PAI-1 There was no significant effect of NAC or placebo on plasma PAI-1 antigen or activity at either $2 \mathrm{~h}$ after the first dose or after 7 days of oral dosing $(p>0.05)$.

Intraplatelet $t G S H$ Intraplatelet $\mathrm{tGSH}$ was not significantly altered in either the NAC or placebo arms of the study at $+2 \mathrm{~h}$ (Fig. 4 a,b). However, there was a clear association between the effect of NAC on tGSH after $2 \mathrm{~h}$ and the baseline pre-NAC tGSH that was not seen in the placebo arm (Fig. 4c). Subsequent subgrouping of patients into those with baseline platelet $\mathrm{tGSH}>120 \mathrm{nmol} / \mathrm{mg}$ protein (' $\mathrm{GSH}$ replete') and those below this cut-off ('GSH deficient'), clearly shows that NAC is only effective in enhancing tGSH in the group that is GSH deficient (Fig. 4d). This effect was not seen in the same subgroupings after placebo (Fig. 4e). There was no association between plasma NAC detection and either change in platelet-monocyte conjugation or intraplatelet tGSH (data not shown).

Association of baseline intraplatelet $t G S H$ or $H b A_{1 c}$ with platelet-monocyte conjugation Platelet-monocyte conjugation was inversely correlated with baseline intraplatelet
Fig. 3 Effect of NAC (a) and placebo (b) on plasma microparticle count after $2 \mathrm{~h}$. There was a significant increase in microparticle count at $2 \mathrm{~h}$ in the placebo arm of the study $(p=0.047$ by paired Student's $t$ test) but a trend towards a reduction $(p=0.09)$ in the NAC arm $(n=14)$. Over a 7 day dosing period (c), there was a significant inhibition of

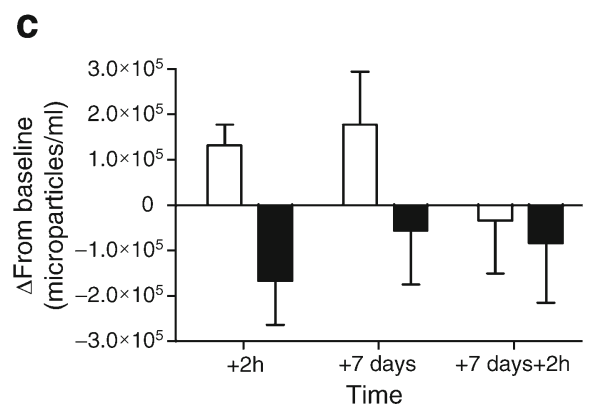

microparticle count between NAC and placebo (black bars, NAC; white bars, placebo: $p=0.016$ for NAC vs placebo; $n=13$ by repeated measures two-way ANOVA) Insufficient blood was drawn from one patient at one visit and data from that patient was excluded from (c) 
a

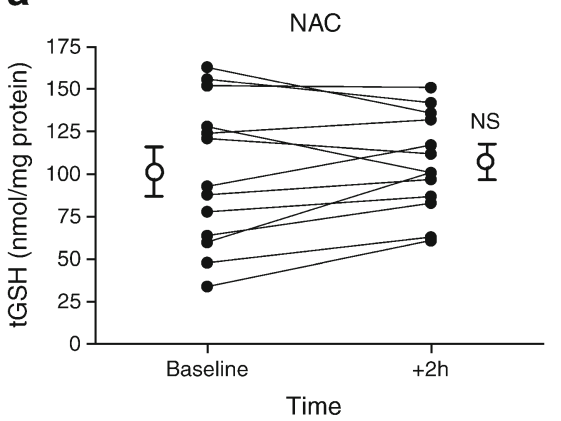

b

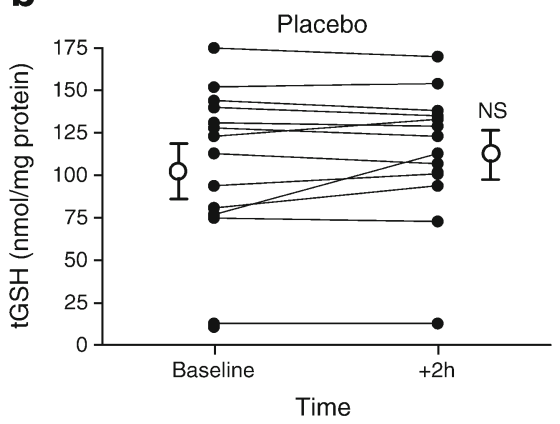

C

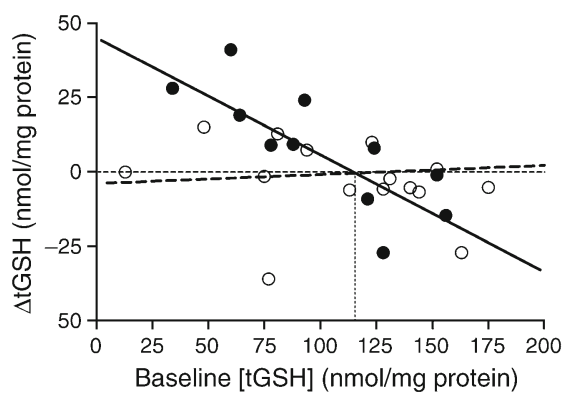

d

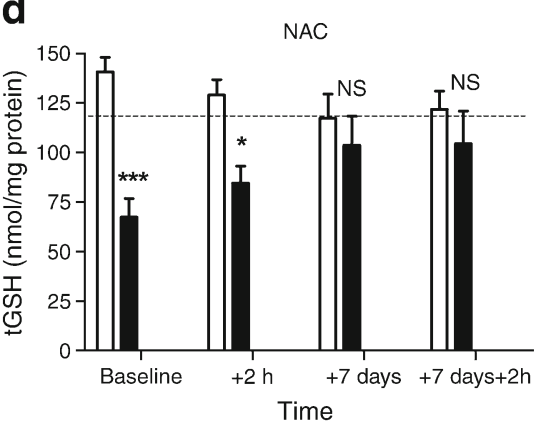

Fig. 4 Effect of NAC on intraplatelet GSH. Neither NAC (a) nor placebo (b) had a significant impact on platelet GSH ( $p>0.05$ for both; $n=12$ ). There was, however, a significant negative correlation between the NAC-induced increase in platelet tGSH at $2 \mathrm{~h}$ and the baseline tGSH (c: black symbols; $\left.p=0.0007, r=0.82, r^{2}=0.66 ; n=12\right)$, which was not reproduced in the placebo arm (white symbols; $p=0.97, r=0.01, r^{2}=$ $0.0002 ; n=12)$. The level at which NAC had no effect was estimated to be $120 \mathrm{nmol} \mathrm{GSH} / \mathrm{mg}$ protein (c: dotted line; this value was used to

tGSH (Fig. 5a), but not with $\mathrm{HbA}_{1 \mathrm{c}}$ (Fig. 5b), in participants in this study.

\section{Discussion}

This double-blind, randomised, placebo-controlled crossover study provides evidence, for the first time, that oral dosing

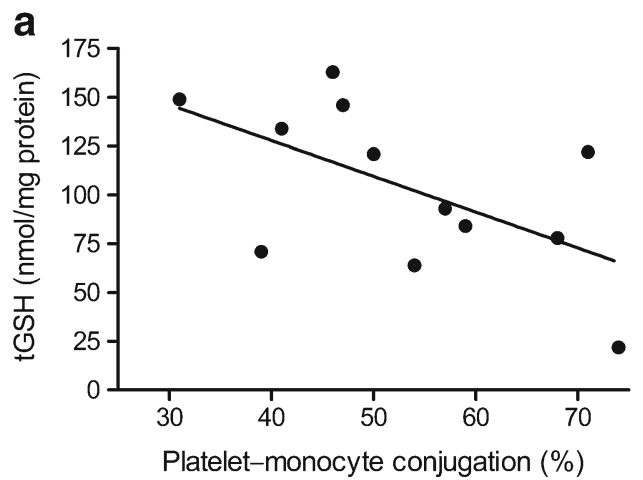

Fig. 5 Correlation between baseline tGSH (a) and $\mathrm{HbA}_{1 \mathrm{c}}$ (b) and platelet-monocyte conjugation (\%). There was a significant negative correlation between tGSH $\left(p=0.047, r=0.58, r^{2}=0.34 ; n=12\right)$ and platelet-monocyte conjugation (a) but no correlation between $\mathrm{HbA}_{1 \mathrm{c}}$

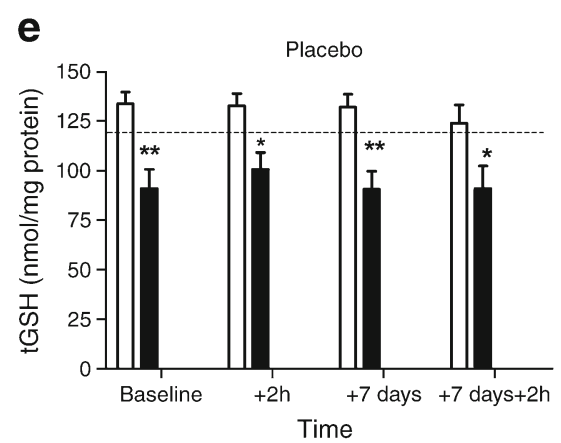

subdivide patients into tGSH replete $(>120 \mathrm{nmol} / \mathrm{mg}$ protein $)$ and deficient $<120 \mathrm{nmol} / \mathrm{mg}$ protein) for further analysis (d, e). Subgroup analysis for individuals with baseline $\mathrm{tGSH}$ of $<120 \mathrm{nmol} / \mathrm{mg}$ protein (black bar; $n=6$ ) and those above this value (white bar; $n=6$ ) clearly showed that GSH is only increased by NAC administration in those with depleted GSH (d: ${ }^{*} p<$ $0.05 ; * * p<0.01 ; * * * p<0.001)$; placebo failed to show the same trend (e) Insufficient sample was available from two patients for GSH analysis

with NAC effectively inhibits platelet-monocyte conjugation and microparticle count in patients with well-controlled type 2 diabetes. The extent of the effect on intraplatelet GSH was inversely correlated with baseline intraplatelet GSH and, unlike $\mathrm{HbA}_{1 \mathrm{c}}$, baseline intraplatelet GSH was also inversely correlated with baseline platelet-monocyte conjugation.

Platelet-monocyte conjugation is gaining recognition as an effective marker for cardiovascular risk [29-32].

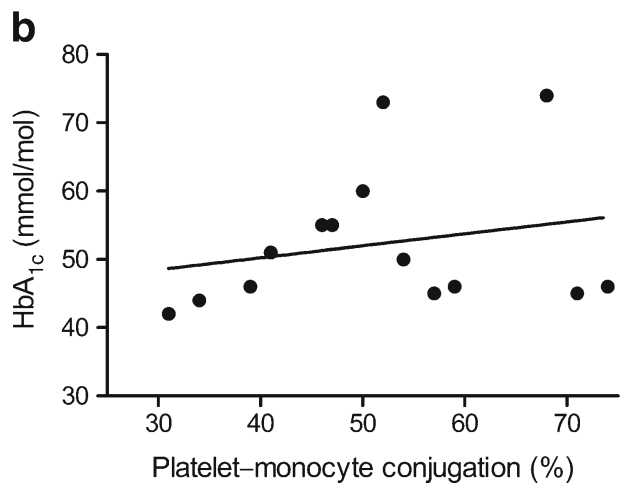

and platelet-monocyte conjugation (b: $p=0.225, R=0.044, r^{2}=0.05$; $n=14$ ). To convert values for $\mathrm{HbA}_{1 \mathrm{c}}$ in $\%$ to $\mathrm{mmol} / \mathrm{mol}$, subtract 2.15 and multiply by 10.929 . Insufficient sample was available from two patients for GSH analysis 
Elevated platelet-monocyte conjugation is indicative of an increased level of circulating platelet activation, which might predispose to thrombus. Moreover, conjugation of activated platelets to monocytes propagates activation of the monocytes themselves [33], increasing the potential for monocyte interaction with the endothelium [34], an early critical event in the atherogenic process [35]. A number of antiplatelet agents [36-38] have been shown to depress platelet-monocyte conjugate formation. Circulating microparticles are cell-derived vesicles that are likewise gaining credence as possible markers of cardiovascular risk because of their elevated levels in a range of cardiovascular conditions [39-43]. While their precise role is still to be fully determined, they appear to be associated with tissue factor and might play a role in thrombosis. On this basis, we selected platelet-monocyte conjugates and microparticles as the principal outcome measures for this study.

Both platelet-monocyte conjugate and microparticle measures are depressed by NAC compared with placebo within $2 \mathrm{~h}$ of administration and the effect is maintained at day 7 following once-daily dosing on the intervening days. The rapidity of onset of the effect on both markers is perhaps surprising, particularly in light of the fact that $\mathrm{NAC}$ is undetectable at the $2 \mathrm{~h}$ time point in 6/14 individuals. However, given the known rapidity of both NAC absorption (time to peak $\left(t_{\max }\right) 0.5-3 \mathrm{~h}$ ) and incorporation into cellular GSH $\left(t_{\max } \sim 1 \mathrm{~h}\right)$, coupled with the wide variability in pharmacokinetics of the drug $\left(t_{1 / 2} 1.4-3.9 \mathrm{~h}\right)$ [20], both the speed of response and the variability in NAC detection at $2 \mathrm{~h}$ are explicable. In the case of plateletmonocyte conjugates, the results indicate that conjugation is a highly dynamic two-way process, the balance of which is disturbed by NAC in favour of disaggregation. With respect to the microparticle data, the results are more complex: at least part of the significant effect of NAC on microparticles is due to prevention or reversal of an increase in microparticle count seen in the placebo arm of the study. The driver for this pro-microparticle effect is unknown, but it might be a simple time-mediated event or could be precipitated by the light breakfast taken by participants between samples. The microparticles measured in this study are not characterised and could therefore be derived from inflammatory or endothelial cells as well as platelets. Further exploratory work is required to identify the driving force behind the change in microparticle effect and how NAC inhibits this process.

Plasma PAI-1 was not affected by NAC at any of the time points measured. PAI-1 was measured because a previous clinical study using injected GSH [13] had found that plasma PAI-1 was reduced after treatment. The inference from our finding is that the effect found previously is specific to plasma GSH as opposed to intracellular GSH. Neither plasma NAC nor the increased intraplatelet tGSH, found in at least a cohort of our test group, significantly influenced PAI-1 antigen or activity.

The impact of oral NAC on intraplatelet tGSH was not as clear cut as we had found previously in vitro [26, 27]. While the tGSH in the placebo arm was reassuringly consistent, that in the NAC arm showed no change in terms of mean value, but revealed a striking pattern of effect that was dependent on the baseline tGSH: low baseline was associated with a large increase, high baseline with either no change or a small decrease. The association was borne out by correlation analysis from which, despite the relatively small sample size, there was found to be a clear inverse correlation between NAC effect size and baseline tGSH, which was not seen in the placebo arm of the study. We used a linear regression plot to estimate the baseline tGSH level that determines likely 'responders' (tGSH deficient) from 'non-responders' (tGSH replete; $>120 \mathrm{nmol} / \mathrm{mg}$ protein). Retrospective subgroup analysis of responders and non-responders confirmed that tGSH was only significantly increased by NAC in the depleted group.

The consequence of this finding is that NAC is only likely to have a biochemical impact in those patients with depleted tGSH (on the evidence of this study, $\sim 50 \%$ of patients with well-controlled type 2 diabetes). The additional finding that there was an inverse association between tGSH and platelet-monocyte conjugation, suggests that tGSH depletion might be an effective marker of cardiovascular risk. Certainly, the correlation between these factors is much clearer than that between $\mathrm{HbA}_{1 \mathrm{c}}$ and platelet-monocyte conjugation, albeit that the patients in this study had relatively well-controlled type 2 diabetes. Furthermore, were low intraplatelet tGSH to be causal in heightened platelet activation in type 2 diabetes, there is the tantalising possibility that NAC administration is most effective in those individuals who are most at risk of cardiovascular complications. This is a novel finding that, if confirmed in a larger study, could point not only to depleted GSH as a contributory factor in heightened platelet activation in type 2 diabetes, but also to the usefulness of intracellular GSH screening before NAC therapy. A critical role for intracellular GSH in determining the extent of NAC benefit might also explain the lack of consistency in results from studies in a range of clinical conditions [15-21], where intracellular GSH has not been a consideration.

In summary, the results of this study indicate that oncedaily oral administration of NAC holds promise in primary prevention of cardiovascular complications associated with type 2 diabetes. These features, together with known benefits with respect to hypertension [24] and endothelial function [23], extend the possible therapeutic targets for NAC 
beyond the confines of type 2 diabetes; patients with metabolic syndrome and other cardiovascular conditions in which oxidative stress and GSH depletion feature might also benefit.

The ability of NAC to reduce platelet-monocyte conjugation is linked to the degree of platelet tGSH depletion, a selective property that might effectively target those at highest risk of cardiovascular complications. In the impending era of personalised medicine, patients most likely to benefit from NAC administration could be identified through platelet tGSH measurement in a blood sample taken before treatment.

A limitation of this study is that it focused on patients with mainly well-controlled type 2 diabetes (mean $\mathrm{HbA}_{1 \mathrm{c}}$ $6.9 \pm 0.9 \%[52.3 \pm 10.3 \mathrm{mmol} / \mathrm{mol}])$. It would therefore be interesting to establish whether similar findings relate to patients with poorer glycaemic control. It is also important to establish whether specific diabetes therapies, such as insulin, impact on platelet-monocyte conjugation, intraplatelet tGSH and the effects of NAC; this would have especially important implications with respect to the relevance of this potential therapy in type 1 diabetes. Finally, the effects have only been determined over a relatively short period ( 8 days). The impact of chronic therapy requires investigating to confirm that the beneficial effects are maintained throughout a much longer intervention period. The study, though relatively small, was adequately powered for the outcome measures.

In conclusion, this study is the first of its kind to show that oral NAC therapy has the potential to reduce cardiovascular risk in those patients with type 2 diabetes who have depleted intraplatelet GSH. There currently exists a paradox in the primary prevention of cardiovascular disease in diabetes: while diabetes is known to increase cardiovascular risk, the principal antiplatelet agent, aspirin, has been found to be ineffective in this patient group and is no longer recommended. This leaves diabetes patients untreated and without an efficacious antiplatelet choice. The current finding is timely in that NAC might represent part of the solution for an unprotected patient population. The results also highlight the importance of individualised therapy for patients with diabetes: NAC may only be effective in that subset of the patient population with type 2 diabetes who are deficient in intracellular GSH. However, the likely effectiveness of the treatment could be screened for in advance through measurement of intraplatelet GSH. In addition, the likely link between oxidative stress, GSH depletion and cardiovascular risk might mean that NAC treatment will target those most vulnerable to cardiovascular disease. An outcome study is now merited to confirm that reduction in platelet-monocyte conjugates is reflected in a reduction in cardiovascular events in this patient group.
Acknowledgements This work was sponsored by NHS Highland and funded by the Chief Scientist Office (CZB/4/622, awarded to I. L. Megson (PI) and S. M. MacRury), Scottish Funding Council, Highlands \& Islands Enterprise and European Regional Development Fund.

Prior presentation at conferences: Diabetes \& Cardiovascular Disease EASD Study Group, 2010; British Pharmacological Society Winter Meeting, 2010; Diabetes UK Annual Professional Conference, 2011.

Duality of interest The authors declare that there is no duality of interest associated with this manuscript.

Contribution statement AT acquired, analysed data and drafted and revised the manuscript. TJW acquired data, reviewed and edited the manuscript. FB, CB and IM acquired data. GFR designed the protocol and edited the manuscript. ID analysed data and edited the paper. SMM and ILM were responsible for the concept and design of the study, analysis of the data and drafting and editing of the paper. All coauthors contributed to and agreed the final draft of this manuscript.

Open Access This article is distributed under the terms of the Creative Commons Attribution Noncommercial License which permits any noncommercial use, distribution, and reproduction in any medium, provided the original author(s) and the source are credited.

\section{References}

1. Ceriello A, Motz E (2004) Is oxidative stress the pathogenic mechanism underlying insulin resistance, diabetes, and cardiovascular disease? The common soil hypothesis revisited. Arterioscler Thromb Vasc Biol 24:816-823

2. Haidara MA, Yassin HZ, Rateb M, Ammar H, Zorkani MA (2006) Role of oxidative stress in development of cardiovascular complications in diabetes mellitus. Curr Vasc Pharmacol 4:215-227

3. Kaneto H, Katakami N, Kawamori D et al (2007) Involvement of oxidative stress in the pathogenesis of diabetes. Antioxid Redox Signal 9:355-366

4. Yu Y, Lyons TJ (2005) A lethal tetrad in diabetes: hyperglycemia, dyslipidemia, oxidative stress, and endothelial dysfunction. Am J Med Sci 330:227-232

5. Houstis N, Rosen ED, Lander ES (2006) Reactive oxygen species have a causal role in multiple forms of insulin resistance. Nature 440:944-948

6. Srivastava S, Joshi CS, Sethi PP, Agrawal AK, Srivastava SK, Seth PK (1994) Altered platelet functions in non-insulin-dependent diabetes mellitus (NIDDM). Thromb Res 76:451-461

7. Schaeffer G, Wascher TC, Kostner GM, Graier WF (1999) Alterations in platelet $\mathrm{Ca}^{2+}$ signalling in diabetic patients is due to increased formation of superoxide anions and reduced nitric oxide production. Diabetologia 42:167-176

8. Ferroni P, Basili S, Falco A, Davi G (2004) Platelet activation in type 2 diabetes mellitus. J Thromb Haemost 2:1282-1291

9. Le Brocq M, Leslie SJ, Milliken P, Megson IL (2008) Endothelial dysfunction: from molecular mechanisms to measurement, clinical implications, and therapeutic opportunities. Antioxid Redox Signal 10:1631-1674

10. Belch J, MacCuish A, Campbell I et al (2008) The prevention of progression of arterial disease and diabetes (POPADAD) trial: factorial randomised placebo-controlled trial of aspirin and antioxidants in patients with diabetes and asymptomatic peripheral arterial disease. BMJ 337:a1840

11. Ogawa H, Nakayama M, Morimoto T et al (2008) Low-dose aspirin for primary prevention of atherosclerotic events in patients with type 2 diabetes: a randomized controlled trial. JAMA 300:2134-2141 
12. Mazzanti L, Mutus B (1997) Diabetes-induced alterations in platelet metabolism. Clin Biochem 30:509-515

13. Martina V, Bruno GA, Zumpano E, Origlia C, Quaranta L, Pescarmona GP (2001) Administration of glutathione in patients with type 2 diabetes mellitus increases the platelet constitutive nitric oxide synthase activity and reduces PAI-1. J Endocrinol Invest 24:37-41

14. Jones AL (1998) Mechanism of action and value of $N$-acetylcysteine in the treatment of early and late acetaminophen poisoning: a critical review. J Toxicol Clin Toxicol 36:277-285

15. Fernandez-Fernandez FJ, Sesma P (2011) Acetylcysteine as adjuvant therapy for vasculitis associated with antineutrophil cytoplasmic antibody. J Rheumatol 38:785

16. Lai KY, Ng WY, Osburga Chan PK, Wong KF, Cheng F (2010) High-dose $N$-acetylcysteine therapy for novel H1N1 influenza pneumonia. Ann Intern Med 152:687-688

17. Virlos IT, Mason J, Schofield D, McCloy RF, Eddleston JM, Siriwardena AK (2003) Intravenous $N$-acetylcysteine, ascorbic acid and selenium-based anti-oxidant therapy in severe acute pancreatitis. Scand J Gastroenterol 38:1262-1267

18. Watt G, Jongsakul K, Ruangvirayuth R (2002) A pilot study of $N$-acetylcysteine as adjunctive therapy for severe malaria. QJM 95:285-290

19. Kramer BK, Hoffmann U (2007) Benefit of acetylcysteine for prevention of contrast-induced nephropathy after primary angioplasty. Nat Clin Pract Nephrol 3:10-11

20. Pendyala L, Creaven PJ (1995) Pharmacokinetic and pharmacodynamic studies of $N$-acetylcysteine, a potential chemopreventive agent during a phase I trial. Canc Epidemiol Biomarkers Prev 4:245-251

21. Aitio ML (2006) $N$-acetylcysteine-passe-partout or much ado about nothing? Br J Clin Pharmacol 61:5-15

22. Pieper GM, Siebeneich W (1998) Oral administration of the antioxidant, $\mathrm{N}$-acetylcysteine, abrogates diabetes-induced endothelial dysfunction. J Cardiovasc Pharmacol 32:101-105

23. Masha A, Brocato L, Dinatale S, Mascia C, Biasi F, Martina V (2009) $\mathrm{N}$-acetylcysteine is able to reduce the oxidation status and the endothelial activation after a high-glucose content meal in patients with type 2 diabetes mellitus. J Endocrinol Invest $32: 352-356$

24. Martina V, Masha A, Gigliardi VR et al (2008) Long-term $\mathrm{N}$ acetylcysteine and L-arginine administration reduces endothelial activation and systolic blood pressure in hypertensive patients with type 2 diabetes. Diabetes Care 31:940-944

25. Anfossi G, Russo I, Massucco P, Mattiello L, Trovati M (2003) Platelet resistance to the antiaggregating effect of $N$-acetyl-L-cysteine in obese, insulin-resistant subjects. Thromb Res 110:39-46

26. Gibson KR, Neilson IL, Barrett F et al (2009) Evaluation of the antioxidant properties of $\mathrm{N}$-acetylcysteine in human platelets: prerequisite for bioconversion to glutathione for antioxidant and antiplatelet activity. J Cardiovasc Pharmacol 54:319-326

27. Gibson KR, Winterburn TJ, Barrett F, Sharma S, Macrury SM, Megson IL (2011) Therapeutic potential of $N$-acetylcysteine as an antiplatelet agent in patients with type-2 diabetes. Cardiovasc Diabetol 10:43
28. Wilkinson IB, Megson IL, MacCallum T et al (2001) Acute methionine loading does not alter arterial stiffness in humans. J Cardiovasc Pharmacol 37:1-5

29. Shoji T, Koyama H, Fukumoto S et al (2005) Platelet-monocyte aggregates are independently associated with occurrence of carotid plaques in type 2 diabetic patients. J Atheroscler Thromb 12:344-352

30. Wang J, Zhang S, Jin Y, Qin G, Yu L, Zhang J (2007) Elevated levels of platelet-monocyte aggregates and related circulating biomarkers in patients with acute coronary syndrome. Int J Cardiol 115:361-365

31. Zhang SZ, Jin YP, Qin GM, Wang JH (2007) Association of platelet-monocyte aggregates with platelet activation, systemic inflammation, and myocardial injury in patients with non-ST elevation acute coronary syndromes. Clin Cardiol 30:26-31

32. Sarma J, Laan CA, Alam S, Jha A, Fox KA, Dransfield I (2002) Increased platelet binding to circulating monocytes in acute coronary syndromes. Circulation 105:2166-2171

33. da Costa MP, van den Berk N, Ulfman LH, Koenderman L, Hordijk PL, Zwaginga JJ (2004) Platelet-monocyte complexes support monocyte adhesion to endothelium by enhancing secondary tethering and cluster formation. Arterioscler Thromb Vasc Biol 24:193-199

34. Ross R (1993) The pathogenesis of atherosclerosis: a perspective for the 1990s. Nature 362:801-809

35. Bournazos S, Rennie J, Hart SP, Fox KA, Dransfield I (2008) Monocyte functional responsiveness after PSGL-1-mediated platelet adhesion is dependent on platelet activation status. Arterioscler Thromb Vasc Biol 28:1491-1498

36. Braun OO, Johnell M, Varenhorst C et al (2008) Greater reduction of platelet activation markers and platelet-monocyte aggregates by prasugrel compared to clopidogrel in stable coronary artery disease. Thromb Haemost 100:626-633

37. Harding SA, Sarma J, Din JN, Maciocia PM, Newby DE, Fox KA (2006) Clopidogrel reduces platelet-leucocyte aggregation, monocyte activation and RANTES secretion in type 2 diabetes mellitus. Heart 92:1335-1337

38. Ray MJ, Walters DL, Bett JN, Cameron J, Wood P, Aroney CN (2005) Platelet-monocyte aggregates predict troponin rise after percutaneous coronary intervention and are inhibited by Abciximab. Int J Cardiol 101:249-255

39. Martinez MC, Tual-Chalot S, Leonetti D, Andriantsitohaina R (2011) Microparticles: targets and tools in cardiovascular disease. Trends Pharmacol Sci 32:659-665

40. Tushuizen ME, Diamant M, Sturk A, Nieuwland R (2011) Cellderived microparticles in the pathogenesis of cardiovascular disease: friend or foe? Arterioscler Thromb Vasc Biol 31:4-9

41. Amabile N, Rautou PE, Tedgui A, Boulanger CM (2010) Microparticles: key protagonists in cardiovascular disorders. Semin Thromb Hemost 36:907-916

42. Shantsila E, Kamphuisen PW, Lip GY (2010) Circulating microparticles in cardiovascular disease: implications for atherogenesis and atherothrombosis. J Thromb Haemost 8:2358-2368

43. Morel O, Toti F, Hugel B et al (2006) Procoagulant microparticles: disrupting the vascular homeostasis equation? Arterioscler Thromb Vasc Biol 26:2594-2604 